\title{
Smart Materials for Biomedical Applications: The Usefulness of Shape-Memory Polymers
}

\author{
Cristiana Fernandes ${ }^{1, a}$, Geetha B. Heggannavar ${ }^{2, b}$, \\ Mahadevappa Y. Kariduraganavar ${ }^{2, \mathrm{c}}$, Geoffrey Mitchell ${ }^{1, \mathrm{~d}}$, Nuno Alves ${ }^{1, \mathrm{e}}$ \\ and Pedro Morouço1, f*
}

\begin{abstract}
${ }^{1}$ CDRSP, Centre for Rapid and Sustainable Product Development, Polytechnic Institute of Leiria, Portugal

2Department of Chemistry, Karnatak University, Dharwad, India

acristiana.fernandes@ipleiria.pt, bgeet.heggannavar@gmail.com ${ }^{\mathrm{C}}$ mahadevappayk@gmail.com, dgeoffrey.mitchell@ipleiria.pt, enuno.alves@ipleiria.pt, fpedro.morouco@ipleiria.pt
\end{abstract}

Keywords: Shape Memory Polymers; 4D Printing; Biomedical Applications; Stimulus.

\begin{abstract}
This review describes available smart biomaterials for biomedical applications. Biomaterials have gained special attention because of their characteristics, along with biocompatibility, biodegradability, renewability, and inexpensiveness. In addition, they are also sensitive towards various stimuli such as temperature, light, magnetic, electro, $\mathrm{pH}$ and can respond to two or more stimuli at the same time. In this manuscript, the suitability of stimuli-responsive smart polymers was examined, providing examples of its usefulness in the biomedical applications.
\end{abstract}

\section{Introduction}

Biopolymers are the polymeric biomolecules, commonly seen as ecological and renewable materials. They present relevant properties, such as biocompatibility, biodegradability and antibacterial activity [1]. Accordingly, smart biomaterials should be able to present self-adaptability; self-sensing; shape-memory, responsiveness; multifunctional; self-repair and decision making, as these features regulate the effects of the external stimulus after they have been printed. [2-7].

Recently, with the emerging interest in the field of smart biomaterials, the term four-dimensional (4D) bioprinting has been proposed. The aim of this technique includes the ability of promoting dynamic changes of the structures and improving the functional response of the construct. It requires stimuli-responsive biomaterials which can be developed, and properly used in the equipment; the best examples of this type of biomaterials are the shape memory polymers [8]. 4D bioprinting offers a great potential for the creation of sophisticated dynamical structures with high resolution, otherwise inaccessible through the 3D bioprinting techniques. Previously, shape-changing polymers were deposited first, and cells were deposited afterwards, which was due to the incompatibility of the methods to their deposition [9].

Shape-memory polymers (SMPs) are a class of polymers that have received deep consideration over the last decade [10]. SMPs are a class of stimuli-responsive materials that demonstrate geometric changes on a temporary shape, according to the application of external stimuli, but are also able to return to their original shape when intended [11,12]. In other words, SMPs are able to "memorize" one or more permanent shapes, that can be kept in temporary shapes, and subsequently, a trigger will lead to remember the permanent shapes $[13,14]$. The stimuli that have been demonstrated to induce changes in shape are temperature; light; magnetic field; electric field and modification of $\mathrm{pH}$ [1].

Temperature-responsive materials can change their shape according to change in temperature. The shape-memory behaviour depends on chemical or physical crosslinks to 3D permanent shape and the glass transition temperature (Tg), to control molecular switching segments that fix the temporal effect. To establish the temporary shape, the material deforms when it is heated above $\mathrm{Tg}$ on the molecular switching segment. When $\mathrm{T}<\mathrm{Tg}$, the temporary shape is obtained and when $\mathrm{T}>\mathrm{Tg}$, the material turns back to its permanent shape [15]. This type of polymers is characterized by a critical solution temperature that may be: a lower critical solution temperature (LCST) or an upper critical solution 
temperature (UCST) $[1,16]$. The LCST can be explicit as the critical temperature in which the polymer solution shows a phase separation from one phase (isotropic state) to two phases (anisotropic state). The hydrophobic and hydrophilic interactions between the polymer chains and the aqueous medium change abruptly within a small temperature range, inducing at the interruption of electrostatic and intermolecular, intermolecular and hydrophobic interactions resulting in chain collapse or expansion [16]. The advantages are: the capacity to deliver hydrophilic and lipophilic drug; sitespecific drug delivery; prevention of toxic organic solvents; and sustained release properties with reduced collateral effects. In contrast, present disadvantages like high-burst drug release, the absence of biocompatibility of the polymeric system and gradual lowering of $\mathrm{pH}$ in the system due to acidic degradation [1].

Light-responsive materials are the polymers which are either sensitive to UV or visible light, depending on the wavelength of the light $[1,11]$. The visible light source is preferred because it is safe and accessible which makes it easy to use [11]. Light-sensitive polymers exhibit some limitations such as inconsistent response due to leaching of non-covalently bound chromophores during swelling or contraction of the system, and a slow response of hydrogel towards the stimulus [1]. The change in shape of these polymers is usually reversible, namely, the freestanding polymer contracts or bends under irradiation, but returns to its original shape once the light source is removed [17].

Magnetic-responsive materials are the polymers that respond to the presence or not of magnetic fields [18]. The principle of this type of shape-memory polymer says that in the presence of a magnetic field gradient, a transactional force will be exerted on the particle/drug complex. This is based on the attraction of magnetic microparticles and nanoparticles to an external magnetic field source [1]. Inorganic magnetic nanoparticles are physically stocked inside or covalently immobilized to a three-dimensional crosslinked network leading to materials with shape and size distortion that occurs reversibly and in the presence of a non-uniform magnetic field [1,18].

Electro-responsive materials depend on their electrically conductive characteristic properties [19] as they transform electric energy into mechanical energy $[1,18]$. In the majority of the cases, this type of biopolymers also exhibits a $\mathrm{pH}$-responsiveness [1]. These materials are relatively hard to design, because the surface must be formed on a conductive electrode to transmit the electrical stimulus, typically gold. One of the advantages of this type of materials and the application of an electric impulse is that it can be less damaging to cells than the average temperature or $\mathrm{pH}$, such as temperature change or $\mathrm{pH}$ change to achieve cellular detachment is minimal in negative articles on cell viability. They also provide an opportunity for collaboration between the fields of cell biology and tissue engineering (TE). Enhanced techniques for creating integrated circuits are also potentially common to a cell culture, allowing a certain degree of control over a cell culture that is difficult to achieve[11].

Similarly, $\mathrm{pH}$-sensitive polymers are the polymers that contain ionizable functional groups that respond to change in $\mathrm{pH}$ [1]. These classes of $\mathrm{pH}$-sensitive systems form a class of stimuli-sensitive materials that apply to biomedical applications [20-22].

Stimuli-responsive materials may also respond to two or more stimuli at the same time.

The health sciences are a priority field of research due to the impact they have on increasing human life expectancy and improving the welfare state by developing advanced effective approaches to address the patient-specific needs [8].

\section{Biomedical Applications of Smart Materials with 3D/4D Bioprinting}

Smart biopolymers that can respond to different external stimuli have a wide range of biomedical applications that include drug delivery, gene delivery, aiming at the treatment of many genetic diseases, and tissue engineering (TE) to regenerate or replace the biologically damaged or diseased tissue or generate replacement organs for a wide range of medical conditions such as heart diseases, diabetes, cirrhosis, osteoarthritis, spinal cord injury and disfiguration [1].

The combination of 3D printing and shape-memory polymers has certain advantages such as the ability to fabricate highly accurate complex structures that can deform in response to different environments. Thus, more biomimetic and multi-functioning biomedical products may be fabricated 
by different techniques and these will have a lot of potential applications in the biomedical field in the future [11].

Similarly, 4D bioprinting has emerged as a beneficial tool for biomedical applications, such as tissue regeneration and drug delivery, due to its essential advantages including the construction of 3D complex tissue based on the responsive materials that are able to reshape or change their functions according to the environmental stimuli; and post-maturation of printed cell populations in scaffolds with programmable architectures, permitting the generation of tissue constructs with functionalities that are similar to those of native tissues [5].

Recently, there has been a significant progress on stereolithography apparatus (SLA) to fabricate SMPs. SLA is one of the most established additive manufacturing (AM) processes and the principal advantage of SLA is producing parts with high resolution and excellent surface finish among all other techniques [23,24]. However, the liquid resins suitable for SLA are limited [25].

A scaffold for TE provides mechanical support, shape, and cell-scale architecture for neo-tissue construction in-vitro or in-vivo as seeded cells expand and organize. It has become progressively apparent for many biomaterial scaffolds which provide more than a temporary architectural structure to a developing tissue construct [26].

There have been a large number of interesting biomedical applications proposed for stimuliresponsive polymer systems, especially in the areas of drug delivery, cell culture surfaces, and diagnostics [27]. Kinetic and thermodynamic control of the stimuli-sensitive response is crucial in all the applications. Therefore, an understanding of the structure-property relationship is essential for further development and rational design of new functional smart materials [28].

Temperature-responsive materials. The thermally induced shape-memory effect has been defined for different materials [29-31], such as polyurethanes [31-35], poly(styrene-blockbutadiene) [31,36] and polynorbornene [31,37,38]; hydrogels [31,39,40]; metallic alloys [31]; and ceramics $[31,41]$. Poly(N-isopropylacrylamide)s (PNIPAAM) is the most prominent candidate as a thermoresponsive polymer [42]. It is a unique material, in view of its biocompatibility and the position of the LCST at $32-33^{\circ} \mathrm{C}$, which make this polymer an ideal candidate for biomedical applications $[11,42-44]$. PNIPAAM copolymers have been studied mainly for the oral delivery of calcitonin and insulin [42]. This material has been utilized for thermoresponsive tissue culture dishes, drug delivery vehicles, haemostasis, and 3D extracellular matrices (ECM) materials [44]. Cytotoxicity tests have already been performed in PNIPAAM by Chastek et al. [45] and Cooperstein et al. [46] they found that the low levels of toxicity have been reported for the cells. PNIPAAM together with poly $(\varepsilon-$ caprolactone) (PCL) is applied for encapsulation or release of cells [5,47,48].

PCL is one of the most extensively investigated degradable polymers [49]. In addition to its inherent biocompatibility, $\mathrm{T}_{\mathrm{m}}$ of PCL can be easily tuned by the variation of its chain length or by copolymerization with other cyclic esters [50]. However, many physical, chemical and biological barriers associated with PCL homopolymers have limited their biomedical applications. For example, PCL is relatively rigid compared to many soft tissues; due to its high crystallinity. It undergoes a plastic deformation when stretched beyond the linear viscoelastic region [49,51]. PCL has proven to show the adhesion and proliferation of human bone marrow-derived stem cells over a period of 3 days $[49,52]$. Poly(N-vinylcaprolactam) (PNVCL) has not been studied as intensively as PNIPAAM, but it also owns very interesting properties for medical and biotechnological applications [42]. PNVCL is known for its superior physiognomies: biocompatibility, solubility, thermosensitive having non-ionic and non-toxic features. Moreover, the LCST of PNVCL $\left(32-34^{\circ} \mathrm{C}\right)$ is near the range of physiological temperature $[53,54]$. It is also considered as a potential thermosensitive material for pharmaceutical applications [55]. Toxicity tests performed by Vihola et al. [56] have not shown any alarming evidence of cytotoxicity in the various tests performed from the time of exposure to temperature.

Poly (N-isopropyl acrylamide) gelatin (PNIPAM-gelatin) was considered by Ohya et al. as a temperature-responsive biodegradable polymer formed in-situ by injectable 3D scaffold [11,44]. Poly (N-ethyl oxazoline) (PEtOx) have a transition temperature around $62^{\circ} \mathrm{C}$, which is too high for drug 
delivery application. Poly(methyl vinyl ether) (PMVE) has a transition temperature in aqueous solution at $37^{\circ} \mathrm{C}$ [42]. Stents based on temperature-responsiveness were some of the initial examples of SMP-based medical devices studied in-vitro and thereby the shape memory of these materials exhibited at body temperature could help to fix a device in the body $[52,57]$.

Films composed of temperature-responsive caprolactone-based SMPs have been shown to support the adhesion and proliferation of mouse fibroblast L929 cells for one week [55]. Stoychev et al. [48] related a design of biodegradable thermoresponsive self-folding capsules that are capable of controlled capture and release of cells. The polymer such as PNIPAM was applied to cell culture [59], targeted protein adsorption [60], control of biomolecular motors [61,62], protein purification [63], photolithography [64] and drug delivery [65]. Yakacki et al [66] explored the PCL for storage, deployment, and subsequent deformation of several SMP stents with respect to their glass transition, crosslinking density, and mechanical design for cardiovascular applications. An ideal choice for a shape-memory medical device is one that stores at room temperature and activates at body temperature [66]. Dadbakhsh et al. [67], focused on nitinol (NiTi) that responds to temperature because they exhibit valuable characteristics, such as good biocompatibility [68-71], low stiffness [72,73], good corrosion resistance, great wear resistance [74-76], high strength, and excellent ductility $[74,77]$.

Light-responsive materials. To study the shape-memory effect, light can be used as an activator to extend the applications of shape-memory polymers especially in the medical devices where the triggers other than heat are highly desirable $[17,78]$. Hydrogels that respond to light are very attractive for biomedical applications, particularly if visible light can be used in the production of devices for ophthalmic drug delivery. Generally, gels respond relatively fast to light as compared to other stimuli [79]. Leidlein et al. [80] proposed a new class of shape-memory polymers activated using light. Similarly, Hearon et al. [12] employed a laser actuated SMP microgripper where the stimulus is UV light with polyurethane (PU). Unger et al. [81] developed the biocompatible hydrogels that can open the new fields of applications such as biotechnology, light-controlled cell growth or light-controlled drug delivery. These hydrogels are known for their dynamic response to swelling. Among the hydrogels, 2-hydroxyethyl methacrylate (HEMA) is widely employed as a shape memory polymer.

Magnetic-responsive materials. Recently, Wen et al. [82] developed magnetic responsive alginate/chitosan hydrogel for cell separation. The potentiality of this was achieved by optimizing the molecular weight of the chitosan, the membrane crosslinking time, and the microcapsule size. Wang [11], demonstrated strategies based on the magnetic response scaffolds for the application of mechanical cues and an innovative methodology for obtaining a suitable prevascularized cardiac mechanism to replace the damaged myocardial tissue. Amaral and Pasparakis [83] developed a matrix with palmitoyl chitosan co-encapsulated with superparamagnetic iron oxide nanoparticles (SPIONs), with improved magnetic properties, and anticancer drug, such as paclitaxel (PTX). They studied how the resulting nanoparticles can serve as biocompatible and biodegradable carriers for the accurate delivery of powerful cytotoxic anticancer agents such as PTX. Similarly, Lin et al. [84] fabricated the alginate microfibers using a modular microfluidic system for controlled drug release with the magnetic response and cell culture.

Electro-responsive materials. This class of materials has recently attracted rapidly expanding the scientific and technological interest due to their potential applications in sensors and actuators, robotics and artificial muscles, optical systems, drug delivery, space, ocean, and energy harvesting applications [85]. Chen et al. [86], developed a multi-functional hybrid microfibers core-shell with a new intelligent biomaterial for biomedical applications. The resulting material promises a flexible model for a reconstruction of electro-sensitive tissues by mimicking the muscle fibres or nerve networks to demonstrate the excellent biocompatibility and electroactivity of microfibers to a cell culture. 
pH-responsive materials. Poly(acrylic acid) (PAAc) and poly(methacrylic acid) (PMAc) are the materials that have the ability to swell by varying the $\mathrm{pH}$ for therapeutic applications $[65,87,88]$. Ethyl acrylic acid (EAAc) and PAAc form $\mathrm{pH}$-sensitive polymers and copolymers that become abruptly hydrophobic as the $\mathrm{pH}$ decreases [27]. Accordingly, $\mathrm{Hu}$ et al. [89], used several polysaccharides, including chitosan, alginate, dextran, hyaluronic acid and their derivatives, and these have $\mathrm{pH}$ sensitive groups in their side chains, which could be employed for drug delivery applications. These polysaccharides are biocompatible, biodegradable, non-toxic in nature and can be easily processed.

Usually, the hydrogels are synthesized from polymers containing weakly acidic (-COOH) [90] or weakly basic $\left(-\mathrm{NH}_{2}\right)$ functional groups [91], the swelling and collapsing processes being the result of protonation/deprotonation of the sensitive moieties [20]. Nadgorny et al. [92] printed a pH-responsive hydrogel valve that validated the dynamic and reversible swelling and shrinkage behaviour that can regulate the flow rate under various $\mathrm{pH}$ levels [93]. Additionally, this structure can serve as a useful platform for $\mathrm{pH}$-responsive membranes [94] and photonic gels [95]. Infections and inflammations can cause an acidic microenvironment, where $\mathrm{pH}$ sensitive scaffolds can swell and lead to increased oxygen penetration and cellular infiltration to increase the tissue regeneration. Micro/nanoparticle systems with $\mathrm{pH}$-responsive properties have also been developed for inflammation treatment. These classes of polymers have the potential for application in cancer immunotherapy [11].

Dual/Triple-responsive materials. Ban et al. reported the dual and triple -responsive SMP that allows the shape deformation when it is irradiation by UV light and stable even in the visible light, and the original shape is recovered at higher temperature [96]. Wu et al. [97] developed UV/heat dual-responsive triple shape memory polymer, which deformed with UV light and returned to original shape with temperature. The NIPAAm and acrylic acid copolymer (AAc) respond to temperature and $\mathrm{pH}$ [27]. According to Hoffman et al. [27], the Dong \& Hoffman oral delivery system is based on an intelligent $\mathrm{pH}$ sensitive and temperature sensitive matrix for acid sensitive and enzymatic drugs. Additionally, a clinical application for oral administration can be found, if the polymer can be shown as non-toxic.

Recently, Chikh Alard et al. [98] prepared the copolymers capable of multi-responsiveness, namely magnetic/light or magnetic/light/pH for biosensors, coatings and controlled drug delivery systems [99]. They used magnetic tetrahalogenoferrate(III) anions along with a polymeric backbone based on poly[2-(N, N-dimethylamino)ethylmethacrylate] (PDMAEMA). Indulekha et al. [100] have developed a system for a therapeutic solution of hyperthermia mediated administration for the treatment of breast cancer, responsive to temperature and $\mathrm{pH}$. This system is composed of PNVCLg-Chitosan polymer loaded with an anticancer drug, doxorubicin hydrochloride (DOX). Electromagnetic actuation appears to have attracted the most interest recently. In this phenomenon, heat is generated through power losses of magnetic filler. Reports have mostly focused on the actuation of SMPs targeting the medical applications. However, in many cases, the field strengths and frequencies largely exceed than those applicable in medical applications [14].

Since the environment-sensitive hydrogels could be developed into bio-inks for 3D bioprinting and affect cell behaviour and differentiation, the materials show excellent potential for clinical applications in the future. Although an insufficiency of reports considering the potential clinical use of self-healing hydrogels, shape-memory polymers, and conductive polymers combined with 3D printing, these smart materials gifted printed scaffolds with smartness as well as influenced cell proliferation and differentiation. Therefore, the clinical applications of the materials might gradually come into focus in the medical field in the near future [11].

\section{Summary}

In the present work, it was possible examine the advancements that are being accomplished through 4D bioprinting. The development and translation of smart materials for 4D bioprinting technology in biomedicine should be based on evidences as well as on the implementation of the key parameters to print [101]. This technique is opening a new door in medical engineering and will serve 
as a toolbox to solve medical problems, taking its advantages in spite of its limitations and challenges $[5,101,102]$.

There is a wide range of properties that can be capably applied in controlled drug delivery, implants, wound, and tissue engineering. Indeed, shape-memory deformation in response to one or more stimuli for biomedical applications, combined with 3D printing technology and other biomanufacturing, can be used as biomimetic actuators or serve as scaffolds for tissue engineering for minimally invasive surgeries. The future clinical success of this methodology will depend on addressing the current challenges of the field [11]. In the future, the combination of 3D printing/fabrication with shape-memory polymers can help in tissue engineering to overcome the existing barriers and achieve new developments of biomimetic and multifunctional scaffolds.

\section{Acknowledgments}

This research work was supported by the Portuguese Foundation for Science \& Technology (FCT) through the Project references CMUP-ERI/TIC/0021/2014 and UID/Multi/04044/2013. In addition, the authors would like to thank Portuguese National Innovation Agency (ANI) through the Project reference POCI-01-0247-FEDER-017963 and European Regional Development Fund (FEDER), through COMPETE2020 under the PT2020 program (POCI-01-0145-FEDER-023423). The authors (MYK and GBH) acknowledge the financial support of UGC, New Delhi under UPE-FAR-I Program [F. No. 14-3/2012 (NS/PE)] and DST, New Delhi under DST-PURSE-Phase-II Program [F. No. SR/PURSE Phase 2/13(G)].

\section{References}

[1] M.Y. Pattanashetti, N. A., Heggannavar, G. B., \& Kariduraganavar, Smart Biopolymers and Their Biomedical Applications, Procedia Manuf. 12 (2017) 263-279.

[2] Z.X. Khoo, J.E.M. Teoh, Y. Liu, C.K. Chua, S. Yang, J. An, K.F. Leong, W.Y. Yeong, 3D printing of smart materials: A review on recent progresses in 4D printing, Virtual Phys. Prototyp. 10 (2015) 103-122.

[3] S.K. Leist, J. Zhou, Current status of 4D printing technology and the potential of light-reactive smart materials as 4D printable materials, Virtual Phys. Prototyp. 2759 (2016) 1-14.

[4] C.A. Mandon, L.J. Blum, C.A. Marquette, 3D-4D printed objects: New bioactive material opportunities, Micromachines 8 (2017).

[5] B. Gao, Q. Yang, X. Zhao, G. Jin, Y. Ma, F. Xu, 4D Bioprinting for Biomedical Applications, Trends Biotechnol. 34 (2016) 746-756.

[6] J. An, C.K. Chua, V. Mironov, A perspective on 4D bioprinting, Int. J. Bioprinting 2 (2016) $3-5$.

[7] S. Wang, J.M. Lee, W.Y. Yeong, Smart hydrogels for 3D bioprinting, Int. J. Bioprinting 1 (2015) 3-14.

[8] P. Morouço, W. Lattanzi, N. Alves. Four-dimensional bioprinting as a new era for tissue engineering and regenerative medicine, Front. Bioeng. Biotechnol. 5 (2017) 61.

[9] A. Kirillova, R. Maxson, G. Stoychev, C.T. Gomillion, L. Ionov, 4D Biofabrication Using Shape-Morphing Hydrogels, Adv. Mater. (2017).

[10] W. Sokolowski, A. Metcalfe, S. Hayashi, L. Yahia, J. Raymond, Medical applications of shape memory polymers, Biomed. Mater. 2 (2007) S23-S27.

[11] Q. Wang, Smart Materials for Tissue Engineering Application, 2017. 
[12] K.L. Hearon, K., Wierzbicki, M. A., Nash, L. D., Landsman, T. L., Laramy, C., Lonnecker, A. T., ... \& Wooley, A processable shape memory polymer system for biomedical applications, Adv. Healthc. Mater. 4 (2015) 1386-1398.

[13] M.D. Hager, S. Bode, C. Weber, U.S. Schubert, Shape memory polymers: Past, present and future developments, Prog. Polym. Sci. 49-50 (2015) 3-33.

[14] P.T. Mather, X. Luo, I.A. Rousseau, Shape Memory Polymer Research, Annu. Rev. Mater. Res. 39 (2009) 445-471.

[15] D.J.M. K. Hearon, M.A. Wierzbicki, L.D. Nash, T.L. Landsman, C. Laramy, A.T. Lonnecker, M.C. Gibbons, S. Ur, K.O. Cardinal, T.S. Wilson, K.L. Wooley, A processable shape memory polymer system for biomedical applications, Adv. Healthc. Mater. 4 (2015) 1386-1398.

[16] W. Cabane, E., Zhang, X., Langowska, K., Palivan, C. G., \& Meier, Stimuli-responsive polymers and their applications in nanomedicine, Biointerphases 7 (2012) 1-27.

[17] H. Jiang, S. Kelch, A. Lendlein, Polymers move in response to light, Adv. Mater. 18 (2006) 1471-1475.

[18] D. Roy, J.N. Cambre, B.S. Sumerlin, Future perspectives and recent advances in stimuliresponsive materials, Prog. Polym. Sci. 35 (2010) 278-301.

[19] Y.-C. Li, Y.S. Zhang, A. Akpek, S.R. Shin, A. Khademhosseini, 4D bioprinting: the nextgeneration technology for biofabrication enabled by stimuli-responsive materials, Biofabrication 9 (2016) 12001.

[20] G. Fundueanu, M. Constantin, S. Bucatariu, P. Ascenzi, pH/thermo-responsive poly(Nisopropylacrylamide-co-maleic acid) hydrogel with a sensor and an actuator for biomedical applications, Polym. (United Kingdom) 110 (2017) 177-186.

[21] C. Liu, Y. Chen, J. Chen, Synthesis and characteristics of pH-sensitive semi-interpenetrating polymer network hydrogels based on konjac glucomannan and poly(aspartic acid) for in vitro drug delivery, Carbohydr. Polym. 79 (2010) 500-506.

[22] M.C. Koetting, N.A. Peppas, PH-Responsive poly(itaconic acid-co-N-vinylpyrrolidone) hydrogels with reduced ionic strength loading solutions offer improved oral delivery potential for high isoelectric point-exhibiting therapeutic proteins, Int. J. Pharm. 471 (2014) 83-91.

[23] Y. Ying, C. Choong, S. Maleksaeedi, H. Eng, J. Wei, P. Su, 4D printing of high performance shape memory polymer using stereolithography, Mater. Des. 126 (2017) 219-225.

[24] F.P.W. Melchels, J. Feijen, D.W. Grijpma, A review on stereolithography and its applications in biomedical engineering, Biomaterials 31 (2010) 6121-6130.

[25] T. Woodfield, K. Lim, P. Morouço, R. Levato, J. Malda, F. Melchels, Biofabrication in Tissue Engineering, in: Ref. Modul. Mater. Sci. Mater. Eng., 2017.

[26] M.E. Furth, A. Atala, M.E. Van Dyke, Smart biomaterials design for tissue engineering and regenerative medicine, Biomaterials 28 (2007) 5068-5073.

[27] A.S. Hoffman, Stimuli-responsive polymers: Biomedical applications and challenges for clinical translation, Adv. Drug Deliv. Rev. 65 (2013) 10-16.

[28] B. Jeong, A. Gutowska, Lessons from nature: Stimuli-responsive polymers and their biomedical applications, Trends Biotechnol. 20 (2002) 305-311.

[29] A. Charlesby, Atomic Radiation and Polymers: International Series of Monographs on Radiation Effects in Materials, 2016.

[30] Y. Kagami, J.P. Gong, Y. Osada, Shape memory behaviors of crosslinked copolymers containing stearyl acrylate, Macromol. Rapid Commun. 17 (1996) 539-543. 
[31] R. Lendlein, A., \& Langer, Biodegradable, Elastic Shape-Memory Polymers for Potential Biomedical Applications, Science (80-. ). 296 (2002) 1673-1676.

[32] B.K. Kim, S.Y. Lee, M. Xu, Polyurethanes having shape memory effects, Polymer (Guildf). 37 (1996) 5781-5793.

[33] J.R. Lin, L.W. Chen, Study on shape-memory behavior of polyether-based polyurethanes. I. Influence of the hard-segment content, J. Appl. Polym. Sci. 69 (1998) 1563-1574.

[34] J.R. Lin, L.W. Chen, Study on Shape-Memory Behavior of Polyether-Based Polyurethanes . II . Influence of Soft-Segment Molecular Weight, (1998) 1575-1586.

[35] T. Takahashi, N. Hayashi, S. Hayashi, Structure and properties of shape-memory polyurethane block copolymers, J. Appl. Polym. Sci. 60 (1996) 1061-1069.

[36] K. Sakurai, Y. Shirakawa, T. Kashiwagi, T. Takahashi, Crystal transformation of styrenebutadiene block copolymer, Polymer (Guildf). 35 (1994) 4238-4239.

[37] K. Sakurai, T. Takahashi, Strain-induced crystallization in polynorbornene, J. Appl. Polym. Sci. 38 (1989) 1191-1194.

[38] K. Sakurai, T. Kashiwagi, T. Takahashi, Crystal structure of polynorbornene, J. Appl. Polym. Sci. 47 (1993) 937-940.

[39] A. Osada, Y., \& Matsuda, Shape memory in hydrogels, Nature 376 (1995) 219.

[40] Z. Hu, X. Zhang, Y. Li, Synthesis and application of modulated polymer gels, Science 269 (1995) 525-527.

[41] M. V. Swain, Shape memory behaviour in partially stabilized zirconia ceramics, Nature 322 (1986) 234-236.

[42] A. Gandhi, A. Paul, S.O. Sen, K.K. Sen, Studies on thermoresponsive polymers: Phase behaviour, drug delivery and biomedical applications, Asian J. Pharm. Sci. 10 (2015) 99-107.

[43] R. Liu, M. Fraylich, B.R. Saunders, Thermoresponsive copolymers: From fundamental studies to applications, Colloid Polym. Sci. 287 (2009) 627-643.

[44] S. Ohya, Y. Nakayama, T. Matsuda, In vivo evaluation of poly(N-isopropylacrylamide) (PNIPAM)-grafted gelatin as an in situ-formable scaffold, J. Artif. Organs 7 (2004) 181-186.

[45] T.T. Chastek, A. Wadajkar, K.T. Nguyen, S.D. Hudson, T.Q. Chastek, Polyglycol-templated synthesis of poly(N-isopropyl acrylamide) microgels with improved biocompatibility, Colloid Polym. Sci. 288 (2010) 105-114.

[46] M.A. Cooperstein, H.E. Canavan, Assessment of cytotoxicity of ( $\mathrm{N}$-isopropyl acrylamide ) and Poly ( N -isopropyl acrylamide ) - coated surfaces, Biointerphases 8 (2013) 1-12.

[47] N.A. Peppas, P. Bures, W. Leobandung, H. Ichikawa, Hydrogels in pharmaceutical formulations, Eur. J. Pharm. Biopharm. 50 (2000) 27-46.

[48] G. Stoychev, N. Puretskiy, L. Ionov, Self-folding all-polymer thermoresponsive microcapsules, Soft Matter 7 (2011) 3277.

[49] X. Yang, C. Cui, Z. Tong, C.R. Sabanayagam, X. Jia, Poly( $\varepsilon$-caprolactone)-based copolymers bearing pendant cyclic ketals and reactive acrylates for the fabrication of photocrosslinked elastomers, Acta Biomater. 9 (2013) 8232-8244.

[50] F. Pilate, R. Mincheva, J. De Winter, P. Gerbaux, L. Wu, R. Todd, J.-M. Raquez, P. Dubois, Design of multi-stimuli responsive shape-memory polymer materials by reactive extrusion, Chem. Mater. 26 (2014) 5860-5867.

[51] M.A. Woodruff, D.W. Hutmacher, The return of a forgotten polymer - Polycaprolactone in the 21st century, Prog. Polym. Sci. 35 (2010) 1217-1256. 
[52] J.G. Hardy, M. Palma, S.J. Wind, M.J. Biggs, Responsive Biomaterials: Advances in Materials Based on Shape-Memory Polymers, Adv. Mater. (2016) 5717-5724.

[53] S.C. Halligan, M.B. Dalton, K.A. Murray, Y. Dong, W. Wang, J.G. Lyons, L.M. Geever, Synthesis, characterisation and phase transition behaviour of temperature-responsive physically crosslinked poly (N-vinylcaprolactam) based polymers for biomedical applications, Mater. Sci. Eng. C 79 (2017) 130-139.

[54] C.C. Ferraz, G.H.C. Varca, J.C. Ruiz, P.S. Lopes, M.B. Mathor, A.B. Lugão, E. Bucio, Radiation-grafting of thermo- and $\mathrm{pH}$-responsive poly(N-vinylcaprolactam-co-acrylic acid) onto silicone rubber and polypropylene films for biomedical purposes, Radiat. Phys. Chem. 97 (2014) 298-303.

[55] J.H. HENNA VIHOLA, ANTTI LAUKKANEN, HEIKKI TENHU, Drug Release Characteristics of Physically Cross-Linked Thermosensitive Poly(N-vinylcaprolactam) Hydrogel Particles, J. Pharm. Sci. 97 (2008) 4783-4793.

[56] H. Vihola, A. Laukkanen, L. Valtola, H. Tenhu, J. Hirvonen, Cytotoxicity of thermosensitive polymers poly(N-isopropylacrylamide), poly(N-vinylcaprolactam) and amphiphilically modified poly(N-vinylcaprolactam), Biomaterials 26 (2005) 3055-3064.

[57] H.M. Wache, D.J. Tartakowska, A. Hentrich, M.H. Wagner, Development of a polymer stent with shape memory effect as a drug delivery system, J. Mater. Sci. Mater. Med. 14 (2003) 109112.

[58] D. Rickert, A. Lendlein, A.M. Schmidt, S. Kelch, W. Roehlke, R. Fuhrmann, R.P. Franke, In vitro cytotoxicity testing of AB-polymer networks based on oligo( $\epsilon$-caprolactone) segments after different sterilization techniques, J. Biomed. Mater. Res. Part B Appl. Biomater. 67B (2003) 722-731.

[59] N. Yamada, T. Okano, H. Sakai, F. Karikusa, Y. Sawasaki, Y. Sakurai, Thermo-responsive polymeric surfaces; control of attachment and detachment of cultured cells, Macromol. Rapid Commun. 11 (1990) 571-576.

[60] B.C. Huber, D. L., Manginell, R. P., Samara, M. A., Kim, B. I., \& Bunker, Programmed Adsorption and Release of Proteins in a Microfluidic Device, Science (80-. ). 301 (2003) 352354.

[61] L. Ionov, M. Stamm, S. Diez, Reversible switching of microtubule motility using thermoresponsive polymer surfaces, Nano Lett. 6 (2006) 1982-1987.

[62] L. Ionov, A. Synytska, S. Diez, Temperature-induced size-control of bioactive surface patterns, Adv. Funct. Mater. 18 (2008) 1501-1508.

[63] B. Galaev, I. Y., \& Mattiasson, "Smart"polymers and what they could do in biotechnology and medicine, Trends Biotechnol. 17 (1999) 335-340.

[64] L. Ionov, S. Diez, Environment-Friendly Photolithography Using Poly ( N isopropylacrylamide ) -Based Thermoresponsive Photoresists, J. Am. Chem. Soc. 131 (2009) 13315-13319.

[65] C. de las H. Alarcón, S. Pennadam, C. Alexander, Stimuli responsive polymers for biomedical applications, Chem. Soc. Rev. 34 (2005) 276-285.

[66] C.M. Yakacki, R. Shandas, C. Lanning, B. Rech, A. Eckstein, K. Gall, Unconstrained recovery characterization of shape-memory polymer networks for cardiovascular applications, Biomaterials 28 (2007) 2255-2263.

[67] S. Dadbakhsh, M. Speirs, J. Van Humbeeck, J.-P. Kruth, Laser additive manufacturing of bulk and porous shape-memory NiTi alloys: From processes to potential biomedical applications, MRS Bull. 41 (2016) 765-774. 
[68] S.E. Bishara, R.D. Barrett, M.I. Selim, Biodegradation of orthodontic appliances. Part II. Changes in the blood level of nickel., Am. J. Orthod. Dentofacial Orthop. 103 (1993) 115-9.

[69] O. Prymak, D. Bogdanski, M. Köller, S.A. Esenwein, G. Muhr, F. Beckmann, T. Donath, M. Assad, M. Epple, Morphological characterization and in vitro biocompatibility of a porous nickel-titanium alloy, Biomaterials 26 (2005) 5801-5807.

[70] A. Bansiddhi, T.D. Sargeant, S.I. Stupp, D.C. Dunand, Porous NiTi for bone implants: A review, Acta Biomater. 4 (2008) 773-782.

[71] R.D. Barrett, S.E. Bishara, J.K. Quinn, Biodegradation of orthodontic appliances. Part I. Biodegradation of nickel and chromium in vitro, Am. J. Orthod. Dentofac. Orthop. 103 (1993) $8-14$.

[72] M.T. Andani, N. Shayesteh Moghaddam, C. Haberland, D. Dean, M.J. Miller, M. Elahinia, Metals for bone implants. Part 1. Powder metallurgy and implant rendering, Acta Biomater. 10 (2014) 4058-4070.

[73] R. Pfeifer, C.W. Müller, C. Hurschler, S. Kaierle, V. Wesling, H. Haferkamp, Adaptable orthopedic shape memory implants, Procedia CIRP 5 (2013) 253-258.

[74] M.H. Elahinia, M. Hashemi, M. Tabesh, S.B. Bhaduri, Manufacturing and processing of NiTi implants: A review, Prog. Mater. Sci. 57 (2012) 911-946.

[75] W. Yan, Theoretical investigation of wear-resistance mechanism of superelastic shape memory alloy NiTi, Mater. Sci. Eng. A 427 (2006) 348-355.

[76] L. Yan, Y. Liu, Effect of temperature on the wear behavior of NiTi shape memory alloy, J. Mater. Res. 30 (2015) 186-196.

[77] K. Otsuka, X. Ren, Physical metallurgy of Ti-Ni-based shape memory alloys, Prog. Mater. Sci. 50 (2005) 511-678.

[78] S. Chaterji, I.K. Kwon, K. Park, Smart polymeric gels: Redefining the limits of biomedical devices, Prog. Polym. Sci. 32 (2007) 1083-1122.

[79] J.F. Mano, Stimuli-responsive polymeric systems for biomedical applications, Adv. Eng. Mater. 10 (2008) 515-527.

[80] R. Lendlein, A., Jiang, H., Junger, O., \& Langer, Light-induced shape-memory polymers, Nature 434 (2005) 879-882.

[81] K. Unger, P. Salzmann, C. Masciullo, M. Cecchini, G. Koller, A.M. Coclite, Novel LightResponsive Biocompatible Hydrogels Produced by Initiated Chemical Vapor Deposition, ACS Appl. Mater. Interfaces 9 (2017) 17408-17416.

[82] H. Wen, T. Gao, Z. Fu, X. Liu, J. xu, Y. He, N. Xu, P. Jiao, A. Fan, S. Huang, W. Xue, Enhancement of membrane stability on magnetic responsive hydrogel microcapsules for potential on-demand cell separation, Carbohydr. Polym. 157 (2017) 1451-1460.

[83] A.J.R. Amaral, G. Pasparakis, Stimuli responsive self-healing polymers: gels, elastomers and membranes, Polym. Chem. (2017).

[84] Y.S. Lin, K.S. Huang, C.H. Yang, C.Y. Wang, Y.S. Yang, H.C. Hsu, Y.J. Liao, C.W. Tsai, Microfluidic synthesis of microfibers for magnetic-responsive controlled drug release and cell culture, PLoS One 7 (2012) 4-11.

[85] T. Manouras, M. Vamvakaki, Field responsive materials: photo-, electro-, magnetic- and ultrasound-sensitive polymers, Polym. Chem. 8 (2017) 74-96. 
[86] C. Chen, X. Chen, H. Zhang, Q. Zhang, L. Wang, C. Li, B. Dai, J. Yang, J. Liu, D. Sun, Electrically-responsive core-shell hybrid microfibers for controlled drug release and cell culture, Acta Biomater. 55 (2017) 434-442.

[87] C. Donini, D.N. Robinson, P. Colombo, F. Giordano, N.A. Peppas, Preparation of poly(methacrylic acid-g-poly(ethylene glycol)) nanospheres from methacrylic monomers for pharmaceutical applications, Int. J. Pharm. 245 (2002) 83-91.

[88] T.R. Kyriakides, C.Y. Cheung, N. Murthy, P. Bornstein, P.S. Stayton, A.S. Hoffman, pHSensitive polymers that enhance intracellular drug delivery in vivo, J. Control. Release 78 (2002) 295-303.

[89] L. Hu, P. Zhang, X. Wang, X. Cheng, J. Qin, R. Tang, pH-sensitive carboxymethyl chitosan hydrogels via acid-labile ortho ester linkage for potential biomedical applications, Carbohydr. Polym. 178 (2017) 166-179.

[90] S.S. Kwon, B.J. Kong, S.N. Park, Physicochemical properties of pH-sensitive hydrogels based on hydroxyethyl cellulose-hyaluronic acid and for applications as transdermal delivery systems for skin lesions, Eur. J. Pharm. Biopharm. 92 (2015) 146-154.

[91] G. Fundueanu, M. Constantin, I. Asmarandei, V. Harabagiu, P. Ascenzi, B.C. Simionescu, The thermosensitivity of $\mathrm{pH} /$ thermoresponsive microspheres activated by the electrostatic interaction of $\mathrm{pH}$-sensitive units with a bioactive compound, J. Biomed. Mater. Res. - Part A 101 A (2013) 1661-1669.

[92] M. Nadgorny, Z. Xiao, C. Chen, L.A. Connal, Three-Dimensional Printing of pH-Responsive and Functional Polymers on an Affordable Desktop Printer, ACS Appl. Mater. Interfaces 8 (2016) 28946-28954.

[93] F. Momeni, S. M.Mehdi Hassani.N, X. Liu, J. Ni, A review of 4D printing, Mater. Des. 122 (2017) 42-79.

[94] M. Orlov, I. Tokarev, A. Scholl, A. Doran, S. Minko, pH-responsive thin film membranes from poly(2-vinylpyridine): Water vapor-induced formation of a microporous structure, Macromolecules 40 (2007) 2086-2091.

[95] Y. Kang, J.J. Walish, T. Gorishnyy, E.L. Thomas, Broad-wavelength-range chemically tunable block-copolymer photonic gels, Nat. Mater. 6 (2007) 957-960.

[96] J. Ban, L. Mu, J. Yang, S. Chen, H. Zhuo, New stimulus-responsive shape-memory polyurethanes capable of UV light-triggered deformation, hydrogen bond-mediated fixation, and thermal-induced recovery, J. Mater. Chem. A Mater. Energy Sustain. 5 (2017) 1451414518.

[97] Y. Wu, J. Hu, C. Zhang, J. Han, Y. Wang, B. Kumar, A facile approach to fabricate a UV/heat dual-responsive triple shape memory polymer, J. Mater. Chem. A 3 (2015) 97-100.

[98] I. Chikh Alard, J. Soubhye, G. Berger, M. Gelbcke, S. Spassov, K. Amighi, J. Goole, F. Meyer, Triple-stimuli responsive polymers with fine tuneable magnetic responses, Polym. Chem. 8 (2017) 2450-2456.

[99] W. Guo, T. Wang, X. Tang, Q. Zhang, F. Yu, M. Pei, Triple stimuli-responsive amphiphilic glycopolymer, J. Polym. Sci. Part A Polym. Chem. 52 (2014) 2131-2138.

[100] S. Indulekha, P. Arunkumar, D. Bahadur, R. Srivastava, Dual responsive magnetic composite nanogels for thermo-chemotherapy, Colloids Surfaces B Biointerfaces 155 (2017) 304-313.

[101] N.J. Castro, C. Meinert, P. Levett, D.W. Hutmacher, Current Developments in Multifunctional Smart Materials for 3D/4D Bioprinting, Curr. Opin. Biomed. Eng. (2017).

[102] A. Kumar, Emergence of Bioprinting in Tissue Engineering: A Mini Review, Adv. Tissue Eng. Regen. Med. Open Access 1 (2016) 1-5. 